\title{
La pronuncia italiana per i giovani apprendenti sloveni: che cosa ne dicono i dizionari? ${ }^{1}$
}

\author{
Italian pronunciation for young Slovenian learners: \\ What do dictionaries say about it?
}

\author{
Robert Grošelj \\ Univerza v Ljubljani \\ robert.groselj@ff.uni-lj.si
}

\begin{abstract}
The article deals with the representation of Italian pronunciation in five contemporary dictionaries for young Slovenian learners. As the use of a dictionary not only stimulates the development of lexical competence, but can also serve as a useful means for pronunciation learning, the article investigates five categories representing phonetic-phonological features in a dictionary: introduction to phonetics/phonology (e.g. a pronunciation guide), phonetic transcription, phonemes, consonant length and accent. The representation of these features in a dictionary for young learners should be clear and coherent, and in some cases a dictionary (especially a dictionary intended for the youngest users) should also feature audio pronunciations. The five dictionaries analysed are fragmentary with regard to the pronunciation: only one dictionary includes audio recordings (although the relation between the spelling and the pronunciation remains unclear, as it does not include a pronunciation guide); two dictionaries include deficient phonological transcriptions and incomplete pronunciation guides; one dictionary contains only the Italian alphabet with corresponding phonemes, while another dictionary is without any elements that could familiarize a Slovenian learner with Italian pronunciation.
\end{abstract}

Keywords: dictionaries for young learners, pronunciation, phonetics, phonology, Italian, Slovenian learners

\footnotetext{
${ }^{1}$ Il presente contributo è stato realizzato nell'ambito del programma di ricerca "Lingua slovena studi di base, contrastivi e applicativi" (P6-0215), finanziato dall'Agenzia pubblica per l'attività di ricerca della Repubblica di Slovenia (ARRS).
} 


\section{INTRODUZIONE}

Le caratteristiche fonetico-fonologiche sono una parte integrante dell'apprendimento e dell' insegnamento L2/LS, come tutti i sottosistemi grammaticali, a prescindere dall'età dell'apprendente. Nonostante questo, nell'insegnamento L2/LS, la pronuncia - a differenza del lessico e di altri aspetti grammaticali - viene spesso trascurata (per l'italiano cfr. Costamagna, 2000, p. 15; Torresan, 2010, p. 69; Schmidt e Pedrazzini, 2016, p. 46; Sisinni, 2016, p. 7; per lo spagnolo cfr. Bartolí Rigol, 2005, p. 3; Torres Martínez, 2017, p. 432; per l'inglese cfr. Tritch Yoshida, 2016, p. 1). La competenza fonologica è spesso ritenuta poco importante, frutto di un'acquisizione inconscia, per l'insegnamento della pronuncia non c'è tempo, i docenti si sentono poco preparati, ecc. (cfr. Torresan, 2010, p. 69; Jones, 2016, p. XI; Schmidt e Pedrazzini, 2016, p. 46; Tritch Yoshida, 2016, p. 1). Nell'insegnamento dell'italiano L2/LS, la posizione marginale della pronuncia è dovuta probabilmente anche alla sua presunta facilità - si afferma che tra la grafia e la pronuncia italiane esista una corrispondenza quasi totale (Costamagna, 2000, p. 15; cfr. simili affermazioni per lo spagnolo e il finlandese in Wells, 1985, p. 45; Bartolí Rigol, 2005, p. 17).

La pronuncia, invece, dovrebbe occupare uno spazio importante nell'insegnamento L2/LS (e non soltanto!), anche quando riguarda gli apprendenti più giovani. Le ragioni per una sua più considerevole inclusione nell'insegnamento L2/LS sono tante: il valore primario dell'input e output orali nella comunicazione linguistica e, soprattutto, nell'insegnamento L2/LS agli apprendenti più giovani; la sua importanza per l'intelligibilità e per la comprensione degli allievi, per lo sviluppo delle abilità di parlato, ascolto e della competenza comunicativa, che influiscono su altre competenze linguistiche; il suo ruolo nello sviluppo della competenza interculturale; un insegnamento più attento della pronuncia può vincere la fossilizzazione linguistica, i blocchi nella competenza comunicativa e nell'apprendimento L2/LS, può vincere e prevenire il transfer negativo (cfr. Bartolí Rigol, 2005, pp. 3-4 e p. 11; Villaescusa Illán, 2009, pp. 127-131; Torresan, 2010, pp. 68-70; Ahmad, 2016, pp. 1-2, p. 5, p. 15; Tritch Yoshida, 2016, p. 1, pp. 5-6; Torres Martínez, 2017, p. 433).

Nell'apprendimento della pronuncia, gli allievi possono contare su diversi elementi didattici - da diverse persone e contenuti a diversi metodi e materiali didattici, tra i quali assumono un ruolo rilevante anche i dizionari (cfr. Yule, 1989; Fraser, 1996; Rivera González, 2005; Godec Soršak, 2015; Ahmad, 2016; Torres Martínez, 2017).

\section{OBIETTIVI E METODOLOGIA}

Il presente contributo si pone come scopo l'analisi della presentazione delle caratteristiche della pronuncia italiana in cinque opere lessicografiche rivolte agli apprendenti più giovani, i cosiddetti dizionari per bambini e ragazzi. Dato il loro 
ruolo nell'apprendimento dell'italiano LS, inclusa la pronuncia (cfr. 4.2), sembra alquanto indispensabile prestare attenzione al loro aspetto fonetico-fonologico.

Dopo una breve presentazione degli aspetti centrali dell'apprendimento/insegnamento della pronuncia L2/LS con particolare enfasi sugli apprendenti più giovani (4) e del ruolo dei dizionari nel suo ambito (4.1), si analizzano alcune caratteristiche fonetico-fonologiche, riguardanti l'italiano, in cinque dizionari per bambini e ragazzi (5). Nell'analisi vengono esaminati gli elementi macrostrutturali, cfr. le osservazioni fonetico-fonologiche riassuntive, le cosiddette guide alla pronuncia (5.1), e quelli microstrutturali - generali come la trascrizione fonetica (5.2), e più specifici (cfr. Nespor, 1993; Costamagna, 2000) come i fonemi italiani (5.3), la durata consonantica (5.4) e l'accento (5.5). Le informazioni fonetico-fonologiche vengono affrontate dal punto di vista della loro utilità, adeguatezza e consistenza. Per la presentazione più dettagliata degli aspetti metodologici si veda il cap. 5 .

\section{CORPUS}

La presente ricerca si basa su cinque dizionari, i.e. 1351BAINS, PIB, 1000IB, OSS e SISS (per i titoli si veda la bibliografia). Osservando il loro contenuto e la loro struttura si possono notare alcune affinità e divergenze (cfr. le categorie in Godec Soršak, 2015, pp. 168-173). Tutti i dizionari sono stampati (PIB è disponibile anche in versione elettronica), contemporanei (pubblicati dopo il 2003), includono (almeno) lo sloveno e l'italiano e si rivolgono agli utenti più giovani (la categoria precisa non è indicata in nessun caso, ma può essere dedotta dalle loro caratteristiche, cfr. la tab. 2 e il cap. 5). Il numero delle unità lemmatiche (incluse le frasi, presentate come lemmi) nei dizionari analizzati varia, da 331 (SISS) a 1267 (1351BAINS). Per la presentazione schematica delle caratteristiche generali si veda la tab. 1.

Tab. 1. Caratteristiche generali dei dizionari analizzati

\begin{tabular}{|l|l|c|l|c|c|}
\hline \multicolumn{1}{|c|}{ dizionario } & \multicolumn{1}{|c|}{$\begin{array}{c}\text { stampato/ } \\
\text { elettronico }\end{array}$} & $\begin{array}{c}\text { data di } \\
\text { pubblicazione }\end{array}$ & $\begin{array}{c}\text { Lingua } \\
\text { lemmatiche }\end{array}$ & $\begin{array}{c}\text { età degli } \\
\text { utenti }\end{array}$ \\
\hline 1351BAINS & stampato & 2003 & $\begin{array}{l}\text { inglese, italiano, tedes- } \\
\text { co, sloveno }\end{array}$ & 1267 & $11-14$ \\
\hline PIB & stampato/elettronico & 2011 & italiano, sloveno & 399 & $6-10 / 11-14$ \\
\hline $1000 I B$ & stampato & 2014 & italiano, sloveno & 927 & $15-18$ \\
\hline OSS & stampato & 2014 & $\begin{array}{l}\text { sloveno, inglese, italia- } \\
\text { no }\end{array}$ & 378 & $6-10$ \\
\hline SISS & stampato & 2018 & sloveno, italiano & 331 & $11-14$ \\
\hline
\end{tabular}


Nei dizionari analizzati, le unità lemmatiche sono organizzate secondo campi semantici, soltanto 1351BAINS è strutturato in ordine alfabetico (con alcuni inserti tematici). In PIB e 1000IB ogni unità lemmatica italiana è seguita dal traducente sloveno, in SISS l'ordine è opposto, in OSS al lemma sloveno seguono i traducenti inglese e italiano, in 1351BAINS, invece, il lemma inglese è seguito dai traducenti italiano, tedesco e sloveno. Le unità lemmatiche e i loro traducenti possono essere scritti con maiuscole (OSS, 1351BAINS) o con minuscole (PIB, 1000IB e SISS). Tutti i dizionari includono materiale illustrativo che sostituisce le definizioni semantiche. Soprattutto SISS e 1000IB (in quantità limitata PIB e OSS) includono frammenti testuali quali frasi, dialoghi et sim.; solo in SISS sono presenti anche esercizi. Nessun dizionario include avvertenze per la consultazione (alcune indicazioni semplici si trovano in SISS, 1000IB e 1351BAINS), mentre quasi tutti i dizionari (tranne OSS) sono dotati di indici alfabetici alla fine. Per la presentazione schematica delle caratteristiche specifiche dei dizionari si veda la tab. 2; per la pronuncia nei dizionari si veda il cap. 5 .

Tab. 2. Caratteristiche specifiche dei dizionari analizzati

\begin{tabular}{|l|l|}
\hline \multicolumn{1}{|c|}{ dizionario } & \multicolumn{1}{|c|}{ caratteristiche specifiche } \\
\hline 1351BAINS & $\begin{array}{l}\text { (1) ordine alfabetico, con inserti tematici; (2) lemma ingl. - traducente it., ted, slov.; } \\
\text { (3) maiuscole; (4) illustrazioni; (5) senza frasi, senza esercizi; (6) indicazioni per la } \\
\text { consultazione semplici; (7) indice slov.-ingl.-it.-ted. }\end{array}$ \\
\hline PIB & $\begin{array}{l}\text { (1) campi semantici; (2) lemma it. - traducente slov.; (3) minuscole; (4) illustrazioni; } \\
\text { (5) con poche frasi, senza esercizi; (6) senza indicazioni per la consultazione; (7) indici } \\
\text { slov. e it. }\end{array}$ \\
\hline 1000IB & $\begin{array}{l}\text { (1) campi semantici; (2) lemma it. - traducente slov.; (3) minuscole; (4) illustrazioni; } \\
\text { (5) con frasi, senza esercizi; (6) indicazioni per la consultazione semplici; (7) indice } \\
\text { slov.-it. }\end{array}$ \\
\hline OSS & $\begin{array}{l}\text { (1) campi semantici; (2) lemma it. - traducente ingl., slov.; (3) maiuscole; (4) illustra- } \\
\text { zioni; (5) con poche frasi, senza esercizi; (6) senza indicazioni per la consultazione; } \\
\text { (7) senza indice. }\end{array}$ \\
\hline SISS & $\begin{array}{l}\text { (1) campi semantici; (2) lemma slov. - traducente it.; (3) minuscole; (4) illustrazioni; } \\
\text { (5) con frasi, con esercizi; (6) indicazioni per la consultazione semplici; (7) indici slov.- } \\
\text { it. e it.-slov. }\end{array}$ \\
\hline
\end{tabular}

\section{APPRENDIMENTO/INSEGNAMENTO DELLA PRONUNCIA L2/LS}

L'apprendimento/insegnamento della pronuncia L2/LS deve - in primo luogo rispettare le caratteristiche, capacità e i bisogni degli allievi. La competenza fonetico-fonologica è la prima competenza, appresa dai bambini, che costruiscono una sorta di "recipiente fonetico" in cui mettono le loro conoscenze lessicogrammaticali (prevale l'input orale). L'apprendimento degli adulti si basa invece su aspetti lessico-grammaticali (l'aspetto fonetico è secondario). La competenza fone- 
tico-fonologica dei bambini è migliore rispetto a quella degli adulti (Bartolí Rigol, 2005, p. 11e 13).

Sull'apprendimento della pronuncia L2/LS non incide solo l'età o lo sviluppo linguistico degli allievi (i giovani imparano la pronuncia in modo più naturale, cfr. l'agilità dell'apparato fonatorio, la fossilizzazione fonologica negli adulti), ma anche la motivazione, l'attitudine, l'esposizione alla L2/LS, i metodi e la qualità dell'insegnamento. Altri elementi che incidono sull'apprendimento della pronuncia L2/LS sono le intereferenze della L1 e la lingua scritta (Skela, 1999, pp. 122-123; Bartolí Rigol, 2005, pp. 13-16, p. 22; Tritch Yoshida, 2016, pp. 3-8).

Secondo la prospettiva comunicativa, l'obiettivo dell'insegnamento della pronuncia non è seguire regole ma apprendere la competenza fonetico-fonetica (Bartolí Rigol, 2005, p. 7). L'obiettivo principale è l'intelligibilità “comoda" (essere compresi, senza dover ripetere spesso gli enunciati), mentre l'accento nativo si sposta su un piano secondario (Bartolí Rigol, 2005, p. 19; cfr. Skela, 1999, p. 126; Schmidt e Pedrazzini, 2016, p. 48) ${ }^{2}$. Tritch Yoshida (2016, p. 2) avverte però che gli allievi dovrebbero avvicinarsi a uno standard riconosciuto (cfr. Rivera González, 2005 , p. 736$)^{3}$; secondo l'autrice bisogna stare attenti sia all'accuratezza che alla scioltezza.

Gli elementi fonetico-fonologici a cui bisogna prestare attenzione includono non solo i fonemi, le loro realizzazioni, la struttura fonetica delle parole (per es. l'accento), ma anche la prosodia (il ritmo e l'intonazione) e i fenomeni fonotattici (assimilazioni, riduzioni et sim.; Skela, 1999, pp. 123-125; Costamagna, 2000; Villaescusa Illán, 2009, p. 132; Tritch Yoshida, 2016, p. 2). L'insegnamento della pronuncia dovrebbe però andare oltre questi fenomeni e coinvolgere i "gruppi di pensiero", la comprensione e la fluidità orale (Ahmad, 2016, p. 15; Bartolí Rigol, 2005, p. 20).

Nella didattica L2/LS, la competenza fonetico-fonologica si costruisce di solito in tre fasi: alla presentazione dell'input orale seguono il recupero e la correzione degli errori (Torresan, 2010, p. 60; cfr. Rivera González, 2005, p. 738). Ogni fase

\footnotetext{
${ }^{2}$ Gli allievi stessi possono preservare il loro accento nativo per varie ragioni: - è una parte della loro identità; - il parlante L1 può essere più tollerante di fronte a un parlante con accento straniero e adattare la propria pronuncia per poter essere capito; - l'allievo non vuole che l'accento nativo influisca sulla sua L1 (cfr. Bartolí Rigol, 2005, p. 19). L'accento straniero (dal punto di vista dei parlanti L2/LS) è un fenomeno praticamente generale (fa parte dell'interlingua degli apprendenti L2/LS) e non dovrebbe creare problemi quando l'espressione è sciolta (Bartolí Rigol, 2005, p. 22). Bisogna dire però che gli apprendenti giovani possono acquisire più facilmente la pronuncia quasi-nativa (Freudstein, 1995, p. 31).

${ }^{3}$ Bartolí Rigol (2005, pp. 21-22), riflettendo sulle varietà LS da insegnare agli apprendenti stranieri, mette in risalto soprattutto le varianti locali quando l'insegnamento della LS avviene in un paese straniero, o dell'insegnante quando l'insegnamento della LS avviene nel paese nativo. La scelta del registro - formale o colloquiale (incluso il registro familiare) - dipende invece dalle necessità degli apprendenti.
} 
può realizzarsi mediante diverse strategie ed esercitazioni - più o meno ludiche, orali e scritte, di transcodificazione (Skela, 1999, pp. 123-125; Torresan, 2010, pp. 60-66). Le attività dovrebbero essere piacevoli, multisensoriali, pratiche, contestualizzate (cfr. l'insegnamento di altre competenze, soprattutto lessicali) e comunicative (Skela, 1999, pp. 122-123; Villaescusa Illán 2009, pp. 133-134; Tritch Yoshida, 2016, p. 9; Torres Martínez, 2017, p. 432) ${ }^{4}$.

Il ruolo centrale in questo processo è svolto dall'insegnante grazie alle sue conoscenze fonetico-fonologiche (conoscendo le caratteristiche delle L1 e L2/LS può prevedere le difficoltà degli allievi) e didattiche (per poter riflettere su contenuti, obiettivi, metodologie adatte; Villaescusa Illán, 2009, p. 132; Bartolí Rigol, 2005, p. 17; Tritch Yoshida, 2016, pp. 8-9). Altre persone che possono influenzare l'apprendimento della pronuncia sono i coetanei, i parlanti L2/LS e i genitori (cfr. Freudstein, 1995, p. 17).

I materiali per l'apprendimento/insegnamento della pronuncia L2/LS, rivolti agli apprendenti più giovani, devono essere interessanti, coinvolgenti, comunicativi, adeguati al livello di conoscenza e alle necessità degli allievi (Villaescusa Illán, 2009, p. 134) e possono includere registrazioni audio e video (Skela, 1999, p. 123; Godec Soršak, 2015, p. 182), manuali con esercizi (Bartolí Rigol, 2005, p. 8; Rivera González, 2005, p. 733; Villaescusa Illán, 2009, p. 133; Torresan, 2010, p. 69; cfr. Costamagna, 1996; Dall'Armellina et al., 2005) e dizionari (cfr. Yule, 1989; Fraser, 1996; Rivera González, 2005; Godec Soršak, 2015; Ahmad, 2016; Torres Martínez, 2017).

\subsection{Dizionari nell'apprendimento/insegnamento della pronuncia L2/LS}

L'uso del dizionario nell'apprendimento/insegnamento L2/LS è essenziale (soprattutto se si tratta degli apprendenti più giovani), dato che stimola lo sviluppo della competenza lessicale (Godec Soršak, 2015, p. 161), ma non solo - i dizionari possono essere anche strumenti fondamentali per l'apprendimento della pronuncia (Rivera González, 2005, p. 733; sul ruolo dei dizionari nella didattica delle lingue si veda per es. Heinz, 2009).

I dizionari per bambini e ragazzi si possono dividere in vari gruppi rispetto all'età dei loro utenti; per la situazione slovena, Godec Soršak (2016, p. 163) propone la divisone in dizionari per bambini (età prescolare), per la scuola primaria (6-10 anni) e quella secondaria (11-14 anni), mentre gli alunni delle scuole secondarie superiori (15-18 anni) dovrebbero saper usare un dizionario monolingue generale.

\footnotetext{
${ }^{4}$ L'insegnamento di una L2/LS rivolto ai bambini dovrebbe basarsi all'inizio esclusivamente sull'interazione tra il suono e l'immagine; in seguito, l'insegnamento della pronuncia dovrebbe diventare un breve, ma costante "spazio ludico" in cui gli allievi possono liberarsi della tensione accumulata durante le fasi dedicate al lessico o alla grammatica (Torres Martínez, 2017, p. 432).
} 
Un dizionario per gli utenti più giovani, in cui non possono mancare le avvertenze per l'uso precise, ma allo stesso tempo comprensibili (accompagnate possibilmente da esercizi e domande), include - come elementi microstrutturali - il lemma (con la struttura sillabica), la parte del discorso, la pronuncia, le forme irregolari, il significato, l'esempio d'uso (qualche elemento può mancare, cfr. però in alcuni dizionari anche elementi ulteriori, per es. l'etimologia, le traduzioni). Molto utili sono le illustrazioni, che aiutano a migliorare l'apprendimento (Godec Soršak, 2015, pp. 164-172; Torres Martínez, 2017, p. 431).

La parte fondamentale di un dizionario in cui sono presentati (in modo riassuntivo) gli aspetti fonetico-fonologici della lingua meta (o la loro rappresentazione lessicografica) è la guida alla pronuncia (cfr. Yule, 1989; Fraser, 1996, p. 235; Rivera González, 2005, p. 735; Godec Soršak, 2015, p. 173). La pronuncia concreta, invece, può essere rappresentata in vari modi - con l'aiuto di simboli fonetici (cfr. l'alfabeto fonetico internazionale, AFI), di segni diacritici o di un sistema di riscrittura (cfr. Wells, 1985, p. 49; Pointon, 2016) ${ }^{5}$. Realizzare un sistema di rappresentazione fonetico-fonologica adatto per gli utenti più giovani è, ovviamente, difficile - normalmente l'AFI e i segni diacritici vengono bollati come troppo difficili, la riscrittura con le "lettere normali" viene considerata un ostacolo per l'apprendimento dell'ortografia corretta (Yule, 1989; Fraser, 1996, p. 222 e 239; Godec Soršak, 2015, p. 182; Torres Martínez, 2017, pp. 430-432). Proprio per questo, i dizionari per bambini (6-10 anni) dovrebbero includere le registrazioni audio (Godec Soršak, 2015, p. 182). In altri casi si consiglia l'uso di una rappresentazione fonologica corretta e coerente (cfr. Rivera González, 2005, pp. 734-735; Godec Soršak, 2015, pp. 175-176) e, quando esistono più varianti di pronuncia, la scelta di una sola possibilità (per l'inglese cfr. Wells, 1985, p. 51). Nella scelta della pronuncia da rappresentare si predilige la variante (o una delle varianti) standard (cfr. Wells, 1985, pp. 45-47).

\section{ANALISI}

L'analisi della presentazione delle caratteristiche fonetico-fonologiche italiane effettuata nei cinque dizionari per bambini e ragazzi comprende cinque categorie (cfr. 4.4). Nella prima vengono analizzate le osservazioni fonetico-fonologiche generali (o introduttive) che aiutano l'utente nella decodifica delle informazioni relative alla pronuncia. Segue l'analisi della trascrizione fonetica con cui si riproduce graficamente la pronuncia italiana. Le ultime tre categorie rappresentano

\footnotetext{
${ }^{5}$ Almeno nel caso dell'inglese, la riscrittura (realizzata sempre con l'alfabeto normale) può essere fonemica o non-fonemica - la prima rappresenta un tipo di trascrizione fonetica, la seconda una riscrittura basata sulle regole ortografiche "normali" (Fraser, 1996, pp. 222-224).
} 
invece tre elementi fonologici centrali, i.e. i fonemi, la durata consonantica e l'accento (Nespor, 1993, pp. 43-70), che vengono analizzati sullo sfondo della "pronuncia neutra dell'italiano" (Canepari, 1999, 2012).

Già uno sguardo superficiale alla presentazione della pronuncia italiana nei cinque dizionari fa notare delle differenze notevoli tra di loro (cfr. la tab. 3) ${ }^{7}$ : tra i dizionari solo PIB include il materiale audio (su CD e su Internet); le note foneticofonologiche si trovano in 1000IB, OSS e SISS (negli ultimi due in misura limitata); la trascrizione fonologica è presente soprattutto in 1000IB e OSS (in SISS in misura limitata), cfr. i foni, la durata consonantica e l'accento (SISS include la trascrizione fonologica dell'alfabeto, in PIB queste informazioni fanno parte delle registrazioni audio) $)^{8}$.

Tab. 3. Pronuncia nei dizionari analizzati

\begin{tabular}{|l|c|c|c|c|c|c|}
\hline dizionario & $\begin{array}{c}\text { materiale } \\
\text { audio }\end{array}$ & $\begin{array}{c}\text { osservazioni } \\
\text { fonetico-fonologiche }\end{array}$ & $\begin{array}{c}\text { trascrizione } \\
\text { fonetico-fonologica }\end{array}$ & foni & durata & accento \\
\hline 1351BAINS & - & - & - & - & - & - \\
\hline PIB & + & - & - & $\varnothing$ & $\varnothing$ & $\varnothing$ \\
\hline 1000IB & - & + & + & + & + & + \\
\hline OSS & - & $(+)$ & + & + & + & + \\
\hline SISS & - & $(+)$ & $(+)$ & $(+)$ & - & - \\
\hline
\end{tabular}

\subsection{Osservazioni fonetico-fonologiche generali}

Le osservazioni fonetico-fonologiche introduttive si riferiscono alle caratteristiche fonetico-fonologiche delle lingue incluse in un dizionario (o alle differenze tra di loro), alla modalità della loro rappresentazione lessicografica oppure al rapporto grafia-pronuncia (come per es. nei dizionari Šlenc, 1997, pp. 9-11; Grad, 1991, pp. 10-11; Mikhailov, 1997, pp. III-V). Tali "guide alla pronuncia”, molto utili

${ }^{6}$ Il concetto della pronuncia italiana neutra (cfr. Canepari, 1999, pp. 19-26) comprende soprattutto 4 tipi di pronuncia: moderna (la più consigliabile; la prima/unica indicata nelle trascrizioni foneticofonologiche), tradizionale, di base toscana/fiorentina (la più consigliata un tempo; indicata dopo il punto, per es. /ع.e/), accettabile, con una certa diffusione nel centro linguistico (abbastanza consigliabile; indicata dopo la virgola, per es. / 8 .e/), e tollerata, con una diffusione limitata nel centro linguistico (meno consigliabile; indicata dopo il punto e virgola, per es. $/ \varepsilon ; \mathrm{e} /$ ).

${ }^{7}$ Il segno + indica la presenza della categoria nel dizionario (le parentesi tonde indicano una presenza limitata), - l'assenza della categoria. Il segno Ø indica che gli elementi analizzati fanno parte delle registrazioni audio.

${ }^{8} \mathrm{~L}$ 'autore delle registrazioni non è indicato né nel dizionario né sul CD. La pronuncia delle registrazioni è neutra - per quel che riguarda la distribuzione dei fonemi, la durata consonantica e l'accento - e non viene analizzata ulteriormente. 
poiché facilitano il passaggio dalla lingua scritta a quella parlata (cfr. Parnwell, 1988, p. 105; Yule, 1989; Rivera González, 2005, p. 735), si trovano in tre dizionari analizzati: in OSS e SISS in misura limitata, 1000IB invece include una nota più ampia.

All'inizio di OSS (cfr. OSS, p. 2) si trova una nota relativa alla pronuncia (per $i$ genitori), dedicata soprattutto alla trascrizione della pronuncia inglese, semplificata, trascritta con l'alfabeto sloveno e senza segni speciali (lo stesso si potrebbe dire per l'italiano); nella nota si aggiunge, inoltre, che in alcuni casi le vocali inglesi e italiane accentate sono sottolineate. L'alfabeto sloveno alla fine del dizionario (OSS, pp. 38-39) non è utile per la pronuncia, cfr. le lettere seguite da parole slovene illustrative, per es. A AVTO, e dai loro traducenti inglesi e italiani con la pronuncia (tra parentesi tonde), per es. CAR (kar), MACCHINA (makkina).

Anche l'alfabeto italiano in SISS (cfr. SISS, pp. 74-75) con le lettere accompagnate dai loro nomi e dai fonemi corrispondenti (trascritti con l'AFI) è poco utile per la consultazione del dizionario, cfr. ad es. I: /i/ o /j/ ì, î $i$; : /ts/o /dz/ zeta.

$1000 \mathrm{IB}$ include alla fine (cfr. 1000IB, p. 132) una nota dedicata alle basi di pronuncia italiana in cui vengono affrontati alcuni grafemi e gruppi grafemici

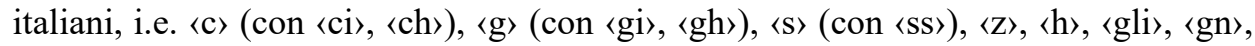
$\langle\mathrm{Sc}\rangle$; la loro realizzazione fonetica è spiegata con l'aiuto delle lettere slovene, per es. la pronuncia di 〈c〉 corrisponde alla pronuncia delle "nostre" $k \mathrm{k}\rangle$ o 〈č (segue un'analisi più dettagliata), 〈h〉 in italiano non si pronuncia, il gruppo 〈gn〉 si pronuncia come 〈nj〉. Il dizionario, purtroppo, non include una spiegazione della trascrizione fonetica adoperata nel dizionario (cfr. 5.2).

\subsection{Trascrizione fonetica}

La trascrizione fonetica è, naturalmente, uno dei mezzi tramite cui si arriva - in un dizionario - alla pronuncia dei singoli lemmi. I sistemi di trascrizione in un dizionario, com'è stato già esposto (cfr. 4.1), possono variare, in generale, dall'AFI all'alfabeto "normale” (Fraser, 1996, pp. 221-225; Godec Soršak, 2015, p. 176). Dei cinque dizionari analizzati solo due ricorrono - in modo consistente - alla trascrizione fonetica o, meglio, fonologica: OSS e 1000IB (in misura molto limitata anche SISS).

La trascrizione semplificata in OSS si basa sull'alfabeto sloveno, cfr. la tab. $4^{9}$. Nella maggioranza dei casi, le trascrizioni dei lemmi (e di alcune frasi, cfr. OSS, p. 2) si trovano tra parentesi tonde e sono scritte con le minuscole (cfr. però le maiuscole nel caso dei titoli), per es. DAI, FORZA, COMINCIAMO... (dai, forca, kominčamo), FIGLIA (filja), IL MERCATO (IL MERKATO). I punti critici della trascrizione, vicina all'utente (perché basata su un sistema di scrittura noto), sono

\footnotetext{
${ }^{9}$ La trascrizione in OSS è indicata dopo il segno $=($ dopo la trascrizione AFI). Cfr. lo stesso sistema anche nella tab. 5 (per la trascrizione in 1000IB).
} 
soprattutto l'assenza dell'accento, i segni che corrispondono a più fonemi, i.e. /e $\varepsilon /=(e)$, $/ \mathrm{o} /=(\mathrm{o}), / \mathrm{u} \mathrm{w} /=(\mathrm{u})$, e i digrammi che rappresentano - in modo troppo semplificato - la pronuncia delle palatali $/ \mathrm{n} /=(\mathrm{nj})$ e $/ K /=(\mathrm{lj}) ; / \mathrm{d} /=(\mathrm{dz})$ e $/ \mathrm{d} \zeta /=(\mathrm{dž})$ sono meno problematici, cfr. 5.3.

Tab. 4. La trascrizione fonologica in OSS

\begin{tabular}{|l|l|l|l|l|l|}
\hline$/ \mathrm{i} /=(\mathrm{i})$ & $/ \mathrm{m} /=(\mathrm{m})$ & $/ \mathrm{p} /=(\mathrm{p})$ & $/ \mathrm{t} /=(\mathrm{c})$ & $/ \mathrm{f} /=(\mathrm{f})$ & $/ \mathrm{r} /=(\mathrm{r})$ \\
$/ \mathrm{e} /=(\mathrm{e})$ & $/ \mathrm{n} /=(\mathrm{n})$ & $/ \mathrm{b} /=(\mathrm{b})$ & $/ \mathrm{d} /=(\mathrm{dz})$ & $/ \mathrm{v} /=(\mathrm{v})$ & $/ \mathrm{l} /=(\mathrm{l})$ \\
$/ \mathrm{g} /=(\mathrm{e})$ & $/ \mathrm{n} /=(\mathrm{nj})$ & $/ \mathrm{t} /=(\mathrm{t})$ & $/ \mathrm{g} /=(\mathrm{c})$ & $/ \mathrm{s} /=(\mathrm{s})$ & $/ \mathrm{h} /=(\mathrm{lj})$ \\
$/ \mathrm{a} /=(\mathrm{a})$ & & $/ \mathrm{d} /=(\mathrm{d})$ & $/ \mathrm{d} /=(\mathrm{d} \mathrm{z})$ & $/ \mathrm{z} /=(\mathrm{z})$ & $/ \mathrm{j} /=(\mathrm{j})$ \\
$/ \mathrm{j} /=(\mathrm{o})$ & & $/ \mathrm{k} /=(\mathrm{k})$ & & $/ \mathrm{J} /=(\mathrm{s})$ & $/ \mathrm{w} /=(\mathrm{u})$ \\
$/ \mathrm{o} /=(\mathrm{o})$ & & $/ \mathrm{g} /=(\mathrm{g})$ & & & \\
$/ \mathrm{u} /=(\mathrm{u})$ & & & & & \\
\hline
\end{tabular}

La trascrizione fonologica in 1000IB, più dettagliata, si basa sull'AFI, cfr. la tab. 5. La pronuncia di tutti i lemmi (e di alcune frasi), trascritta con l'AFI (l'accento fa parte della trascrizione), è indicata tra parentesi quadre (in corsivo), per es. la mensola ['mensola], Sono le sette ['ssno 'le 'sette], benché la trascrizione fonologica preveda barre oblique (che appaiono due volte, per es. il ghiacciolo lghjat'fflo/). I punti problematici della trascrizione (in generale) sono la presenza degli allofoni nasali [ $\mathrm{y} \mathrm{m}$ n] , trascritti con [ $\mathrm{y} \mathrm{n}$ ] (si veda la tab. 7), e l'assenza di una nota esplicativa riguardante l'AFI. In SISS (cfr. SISS, 74-75) si trovano le lettere dell'alfabeto italiano, accompagnate dai fonemi corrispondenti trascritti con l'AFI.

Tab. 5. La trascrizione fonologica in 1000IB

\begin{tabular}{|c|c|c|c|c|c|}
\hline $\begin{array}{l}/ \mathrm{i} /=[\mathrm{i}] \\
/ \mathrm{e} /=[\mathrm{e}] \\
/ \mathrm{\varepsilon} /=[\mathrm{\varepsilon}] \\
/ \mathrm{a} /=[\mathrm{a}] \\
/ \mathrm{o} /=[\mathrm{o}] \\
/ \mathrm{o} /=[\mathrm{o}] \\
/ \mathrm{u} /=[\mathrm{u}]\end{array}$ & $\begin{array}{l}/ \mathrm{m} /=[\mathrm{m}] \\
/ \mathrm{n} /=[\mathrm{n}] \\
/ \mathrm{n} /=[\mathrm{n}] \\
{[\mathrm{n}]=[\mathrm{y}]} \\
{[\mathrm{m}]=[\mathrm{n}]} \\
{[\mathrm{n}]=[\mathrm{y}]}\end{array}$ & $\begin{array}{l}/ \mathrm{p} /=[\mathrm{p}] \\
/ \mathrm{b} /=[\mathrm{b}] \\
/ \mathrm{t} /=[\mathrm{t}] \\
/ \mathrm{d} /=[\mathrm{d}] \\
/ \mathrm{k} /=[\mathrm{k}] \\
/ \mathrm{g} /=[\mathrm{g}]\end{array}$ & $\begin{array}{l}/ \mathrm{ts} /=[\mathrm{ts}] \\
/ \mathrm{dz} /=[\mathrm{d}] \\
/ \mathfrak{t} /=[\mathrm{tg}] \\
/ \mathrm{d} /=[\mathrm{d}]\end{array}$ & $\begin{array}{l}/ \mathrm{f} /=[\mathrm{f}] \\
/ \mathrm{v} /=[\mathrm{v}] \\
/ \mathrm{s} /=[\mathrm{s}] \\
/ \mathrm{z} /=[\mathrm{z}] \\
/ \mathrm{S} /=\left[\int\right]\end{array}$ & $\begin{array}{l}/ \mathrm{r} /=[\mathrm{r}] \\
/ 1 /=[1] \\
/ \mathrm{K} /=[\mathrm{K}] \\
/ \mathrm{j} /=[\mathrm{j}] \\
/ \mathrm{w} /=[\mathrm{w}]\end{array}$ \\
\hline
\end{tabular}

\subsection{Fonemi (foni) dell'italiano}

La trascrizione fonologica consente all'utente di informarsi sulla pronuncia di parole o, meglio, sui loro elementi distintivi (per es. sui fonemi, sulla durata consonantica e sull'accento). Il sistema fonologico italiano è costituito da 7 vocali /i e $\varepsilon$

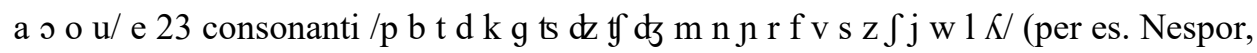


1993, p. 61; Graffi e Scalise, 2008, pp. 79-81; Bertinetto, 2010, pp. 7-10), mentre il sistema sloveno include 8 vocali /i e $\varepsilon$ a $\rho$ o u ə/ e 22 consonanti /p b t d k g ts d t ds m n r f s z J 3 x v j 1/ (Toporišič, 2004, p. 48 e 85; Tivadar e Jurgec, 2003, pp. 214-216); per gli sloveni sono problematiche soprattutto le vocali italiane /e $\varepsilon \circ \mathrm{o} /$, a causa della loro distribuzione, dell'incongruenza grafica e della non-differenziazione di questi fonemi in molti dialetti sloveni, e le consonanti $/ \mathrm{n} K \mathrm{r} \mathrm{w}$ v/ assenti in sloveno (cfr. Grošelj, 2013; Brecelj, 2013) ${ }^{10}$. Nei dizionari dotati della trascrizione fonologica $\mathrm{i}$ fonemi italiani sono rappresentati in modo spesso incoerente o erroneo.

In OSS, le vocali italiane più problematiche sono, comprensibilmente, $/ \varepsilon \mathrm{s} /: / \varepsilon /$ moderna è sempre (in 71 casi) trascritta con (e) (in 10 casi (e) corrisponde a /e/ accettabile $(/ \varepsilon$.e/), in 11 casi a /e/ tollerata $(/ \varepsilon ; \mathrm{e} /))$; / / / moderna è in tutti i casi (42) trascritta con (o) (una volta /o/ è tollerata $(/ \mathrm{o} ; \mathrm{o} /)$ ). Le vocali /i a/ sono meno critiche: la prima è 5 volte trascritta con $(\mathrm{j})$, la seconda 1 volta con (o). Le consonanti sono rispetto alle vocali - meno difettose: il punto più discutibile rappresenta il fonema $/ \mathrm{w} /$, 15 volte trascritto con $(\mathrm{u})$ e 1 volta con (v) (la possibilità tradizionale $(/ \mathrm{w} . \mathrm{v} /)$ ); seguono /j/, 14 volte trascritta con (i), e /dz/, 4 volte rappresentata da (c) (3 volte si tratta della realizzazione tradizionale $(/ \mathrm{dz} . \mathrm{ts} /))$ e 2 volte da $(\mathrm{z}) ; / \mathrm{z} /$ moderna è 2 volte trascritta con (s) (entrambe le volte corrisponde a /s/ tradizionale (/z.s/)), mentre a /ts/ 1 volta corrisponde $(\mathrm{z})$, a $/ \mathrm{v} /$ in 1 caso (u). In 2 casi il segno (j) risulta superfluo, cfr. ZIO (cijo). I fonemi $/ \Lambda \mathrm{j} /$ sono sempre (10 e 14 volte, rispettivamente) trascritti con (lj) e (nj). Per i punti critici della trascrizione in OSS si veda la tab. $6^{11}$.

Tab. 6. Fonemi in OSS - punti critici

\begin{tabular}{|c|c|}
\hline Vocali & consonanti \\
\hline $\begin{array}{l}/ \mathrm{i} /=(\mathrm{j})^{5(3 \%)}(\text { djarjo }) \\
/ \varepsilon /=(\mathrm{e})^{50(70 \%)}(\text { spekkjo) } \\
-/ \varepsilon, \mathrm{e} /=(\mathrm{e})^{10(14 \%)} \text { (piede) } \\
-/ \varepsilon ; \mathrm{e} /=(\mathrm{e})^{11(15 \%)} \text { (indženjere) } \\
/ \mathrm{a} /=(\mathrm{o})^{1(0,3 \%)} \text { (uoter) } \\
/ \mathrm{J} /=(\mathrm{o})^{41(98 \%)}(\text { orolodžo) } \\
-/ \mathrm{o} ; \mathrm{o} /=(\mathrm{o})^{1(2 \%)} \text { (forbiči) }\end{array}$ & 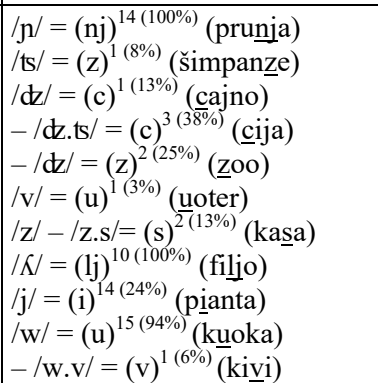 \\
\hline
\end{tabular}

${ }^{10}$ Il fonemi palatali /n $\mathrm{K} /$ sono spesso realizzati come nessi [nj lj], il vibrante /r/ come vibrato [c], il costrittivo /v/ come approssimante (semicostrittivo) [v]; talvolta anche all'approssimante /w/ (cfr. soprattutto la sequenza $/ \mathrm{kw} /$ ) corrisponde la realizzazione [v] (Canepari, 2006a, pp. 205-206; Brecelj, 2013, pp. 196-197).

${ }^{11}$ In apice sono indicati il numero delle occorrenze e la percentuale delle trascrizioni scorrette. 
In generale, la trascrizione in 1000IB è più precisa rispetto a quella in OSS, vi esiste però una variazione più grande. Le vocali più critiche in $1000 \mathrm{IB}$ (cfr. la tab. 7) sono /e $\varepsilon$ o o/: /e/ moderna è trascritta 10 volte con $[\varepsilon]$ (2 volte $[\varepsilon]$ corrisponde a / $\varepsilon /$ tradizionale $(/ \mathrm{e} . \varepsilon /)$, in 1 caso a / $/ \varepsilon /$ accettabile $(/ \mathrm{e}, \varepsilon /))$; la trascrizione $\mathrm{di} / \varepsilon /$ moderna è ancora più variabile $-/ \varepsilon /$ è 23 volte trascritta con [e] (in 2 casi [e] corrisponde a /e/ tradizionale $(/ \varepsilon . \mathrm{e} /)$, in altrettanti casi a /e/ accettabile $(/ \varepsilon, \mathrm{e} /)$ ed /e/ tollerata $(/ \varepsilon ; \mathrm{e} /))$, 2 volte con [ej] (cfr. le possibilità accettabili sarebbero /ei/ o / $/ \mathrm{i}$,ei/) ${ }^{12}, 1$ volta con [wet], [a] (accettabile) e [œ] (la possibilità accettabile sarebbe [ø]);/// moderna è trascritta con [o] 16 volte ( 2 volte [o] corrisponde a /o/ accettabile); /o/ moderna è trascritta con [o] 10 volte. Le altre vocali sono meno problematiche: /i/ è 2 volte trascritta con [j] (in 1 caso / $\mathrm{j} /$ sarebbe accettabile), mentre a / $\mathrm{u} / 1$ volta corrispondono [ou] e [ow] (cfr. nell'ultimo caso la possibilità accettabile intenzionale /o/). Gli errori di trascrizione riguardanti le consonanti in 1000IB sono per lo più individuali, cfr. /d/ trascritta con [t], /g/ con [ts], / $/$ con [sh], /3/ (xenofonema) con [d] , /W/ moderna con $[\mathrm{v}]$ (/v/ è la possibilità tradizionale); cfr. però /dz/ moderna 3 volte trascritta con [ts] (/ts/ sarebbe la possibilità tradizionale in tutti e tre $\mathrm{i}$ casi), /n/ 2 volte trascritta con [n], /s/ 2 volte trascritta con $[\mathrm{z}], / \mathrm{z} /$ moderna 3 volte trascritta con $[\mathrm{s}]$ ( 2 volte $[\mathrm{s}]$ corrisponde a /s/ tradizionale) e, alla fine, $/ \mathrm{j} /$ ben 5 volte trascritta con [i].

Tab. 7. Fonemi (foni) in 1000IB - punti critici

\begin{tabular}{|c|c|}
\hline Vocali & Consonanti \\
\hline 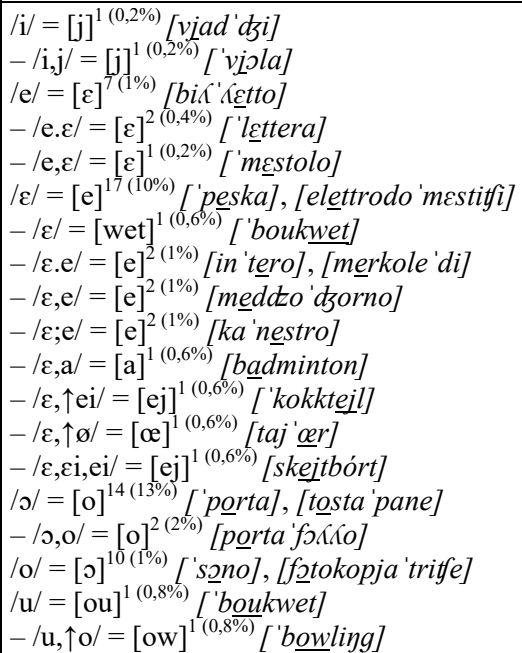 & 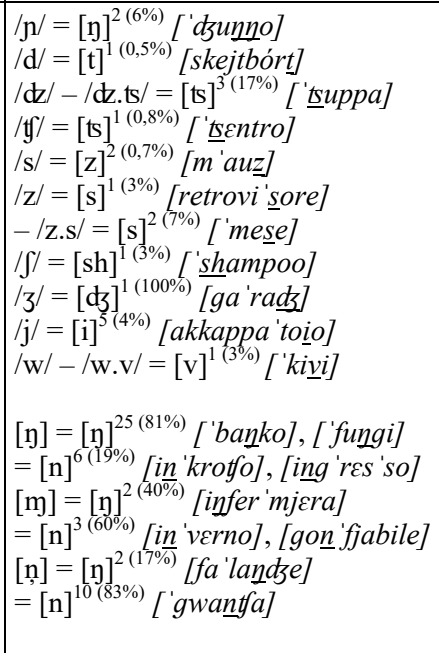 \\
\hline
\end{tabular}
1999).

${ }^{12}$ Il segno $\uparrow$ nella trascrizione indica la pronuncia intenzionale, usata "per fare sfoggio" (Canepari, 
1000IB include - in modo incoerente - anche gli allofoni nasali [n $\mathrm{m} \mathrm{n}$ ]: a [n] velare corrispondono sia [y] (25) che [n] (6), e lo stesso si può dire anche di [m] labiodentale, cfr. [y] (2) e [n] (3), e di [n] postalveopalatale, cfr. [y] (2) e [n] (10). Tra i grafemi superflui, invece, [h] (per es. [pot'tsayghera]) compare 9 volte (in 4 prestiti, come per es. in ['haygar], potrebbe rappresentare la pronuncia tollerata);

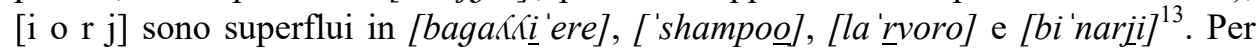
[J] e [k] "ridondanti" si veda il cap. 5.4.

\subsection{Durata consonantica}

La durata consonantica con valore distintivo esiste in italiano, ma non in sloveno, cfr. la differenza tra it. pala 'badile, vanga' e palla 'pallone' (Nespor, 1993, pp. 67-68; Graffi e Scalise, 2008, p. 104; Toporišič, 1992, pp. 25-26; Mikhailov, 1997, p. XV) ${ }^{14}$. In italiano, più precisamente, la durata distintiva riguarda 15 fonemi consonantici (geminabili), i.e. /p b t d k g t d $\mathrm{m} \mathrm{n} \mathrm{r} \mathrm{f} \mathrm{v} \mathrm{s} 1 /$, che possono essere semplici o lunghi; i rimanenti 8 fonemi consonantici hanno durata prevedibile dal contesto fonico: / $\mathrm{z} \mathrm{j}$ w/ sono brevi (ageminabili), mentre /ts d $\int \mathrm{j} K /$ (autogeminanti) hanno durata intrinseca - sono lunghi tra due vocali o tra una vocale e $[\mathrm{j} w]$ (Mioni, 1993, pp. 116-118; Canepari, 1999, pp. 162-164; Bertinetto, 2010, pp. 11-12) ${ }^{15}$. La durata consonantica viene indicata in due dizionari analizzati - in OSS e 1000IB.

Tab. 8. Durata consonantica in OSS

\begin{tabular}{|c|c|}
\hline consonanti geminabili & consonanti autogeminanti \\
\hline 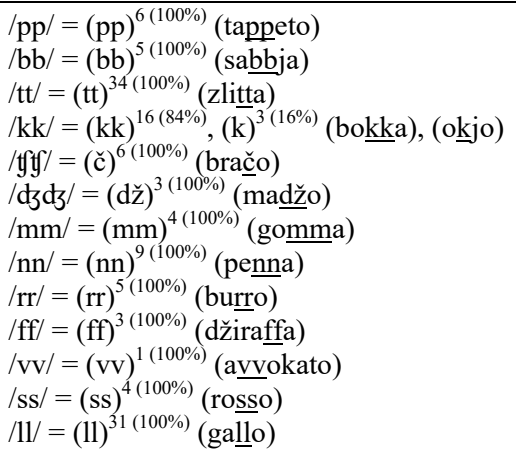 & $\begin{array}{l}/ \mathrm{nj} /=(\mathrm{nj})^{14(100 \%)}(\text { prunja }) \\
/ \mathrm{tsts} /=(\mathrm{c})^{4(80 \%)},(\mathrm{cc})^{1(\overline{20} \%)}(\text { ragaco }),(\text { strucco }) \\
/ \iint /=(\breve{s})^{6(100 \%)}(\text { koša }) \\
/ \Lambda K /=(1 \mathrm{j})^{10(100 \%)}(\text { filljo) }\end{array}$ \\
\hline
\end{tabular}

${ }^{13} \mathrm{La}$ trascrizione fonetico-fonologica non dovrebbe includere apostrofi (3 volte), per es. ['sala d'a 'spetto].

${ }^{14} \mathrm{La}$ durata vocalica in italiano, non distintiva, è predicibile dal contesto fonico (Nespor, 1993, p. 68; Graffi e Scalise, 2008, p. 104). Per una riflessione più approfondita si veda Canepari (1999, pp. 164-165).

${ }^{15}$ I parlanti sloveni spesso non producono né la geminazione né l'autogeminazione (Canepari, 2006a, p. 206; Brecelj, 2013, p. 197). 
La trascrizione delle consonanti lunghe (geminabili e autogeminanti) in OSS risulta lacunosa (per gli esempi si veda la tab. 8). Le consonanti geminabili lunghe $/ \mathrm{p} \mathrm{b} \mathrm{t} \mathrm{m} \mathrm{n} \mathrm{r} \mathrm{f} \mathrm{v} \mathrm{s} 1 /$ sono trascritte correttamente in tutti i casi; / $/ \mathrm{f} \mathrm{d} /$ lunghe sono sempre trascritte come semplici, mentre $/ \mathrm{kk} /$ è trascritta come lunga in 16 casi, come semplice in 3 casi (in OSS / $\mathrm{d} /$ lunghe non appaiono). Le consonanti autogeminanti /ts $\int \mathrm{n} K /$ sono per lo più trascritte erroneamente, i.e. come semplici, solo /ts/ è trascritta una volta come lunga (/dz/ non appare in posizione rilevante per la durata).

La maggioranza delle consonanti geminabili lunghe in 1000IB è trascritta correttamente (per gli esempi si veda la tab. 9) - /p t t $\mathrm{m} \mathrm{n} \mathrm{r} \mathrm{f} \mathrm{s/} \mathrm{lunghe} \mathrm{sono} \mathrm{sempre}$ trascritte correttamente, mentre $/ \mathrm{b}$ ds $1 /$ lunghe sono trascritte erroneamente (come semplici) solo 1 volta; $/ \mathrm{k} /$ lunga è trascritta correttamente 39 volte, 1 volta [kk] corrisponde alla possibilità accettabile $(/ \mathrm{k}, \mathrm{kk} /$, in un prestito), una volta (di nuovo in un prestito) a una $/ \mathrm{k} /$ semplice, /d/ lunga è trascritta come lunga 1 volta, come semplice 2 volte (in due abbreviazioni; /g v/lunghe non appaiono in 1000IB). L'unica consonante autogeminante trascritta correttamente sempre è $/ \mathrm{K} / ; \mathrm{nj} /$ è trascritta bene 15 volte, 1 volta è trascritta come [ỵ] (cfr. 5.3); /tsts/ è trascritta come lunga 13 volte, come semplice 8 volte; / $/$ lunga è trascritta correttamente 11 volte, 9 volte [J] corrisponde a una $/ \int /$ semplice in posizione iniziale di parola; /dzç/ è rappresentata con [ddz] 4 volte, 1 volta alla /dzcz/ moderna corrisponde [z] (accettabile).

Tab. 9. Durata consonantica in 1000IB

\begin{tabular}{|c|c|}
\hline consonanti geminabili & consonanti autogeminanti \\
\hline 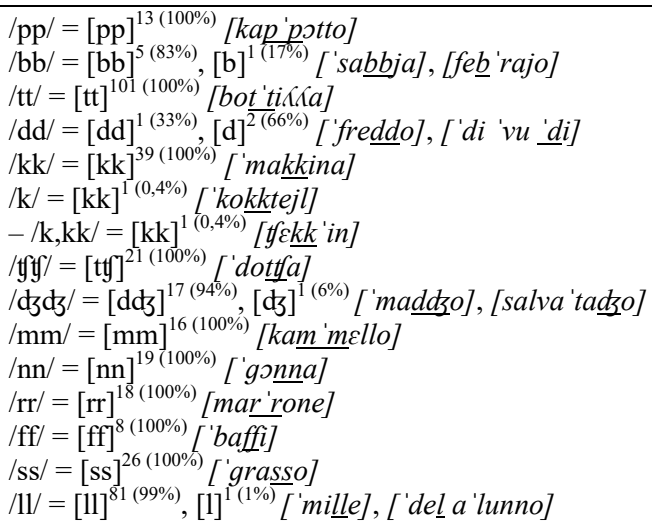 & 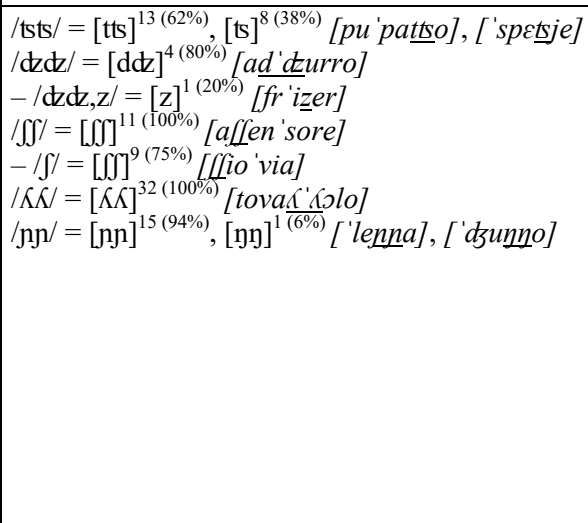 \\
\hline
\end{tabular}

\subsection{Accento}

Sia in italiano che in sloveno l'accento - foneticamente determinato da maggiore forza o intensità articolatoria su una sillaba (Toporišíč, 1992, p. 116; Nespor, 1993, p. 64; Canepari, 1999, pp. 148-149; Graffi e Scalise, 2008, p. 105) - 
è mobile e distintivo, cfr. it. /'ankora/ 'ormeggio' (sost.) e /an'kora/ 'fino a ora/allora' (avv.) (Nespor, 1993, p. 65; Canepari, 1999, pp. 149-150; Graffi e Scalise, 2008, p. 105) e slov. /'govori/ 'discorsi' (sost.), /go'vori/ 'parla' (imper., 2a sg.), /gэvo'ri/ 'parla' (ind., 3a sg.) (Greenberg, 1987, p. 177; Toporišič, 2004, p. 64) ${ }^{16}$. Nella maggior parte dei casi, gli apprendenti sloveni imparano l'accento di ogni singolo elemento lessicale italiano, esistono però dei contesti morfologici (alcune forme flesse o derivate) in cui la posizione dell'accento è prevedibile (cfr. anche l'accento grafico; Canepari, 1999, pp. 124-128, pp. 150-152; Graffi e Scalise, 2008, p. 105; Bertinetto, 2010, p. 15). Inoltre bisogna menzionare che l'accentazione più frequente in italiano è quella piana - è piana la metà delle parole italiane (Canepari, 1999, p. 150) o, perfino, il 75\% delle parole di largo uso (Palermo, 2015, p. 34). Tra i dizionari analizzati solo 1000IB e OSS includono informazioni sull'accento (l'ultimo in misura molto limitata).

In OSS, nella nota sulla pronuncia, viene precisato che in alcune parole inglesi e italiane le vocali accentate sono sottolineate (cfr. OSS, p. 2). Per l'italiano questo succede solo nel caso banale del lemma piano SCOLARI (skolari). Data la prevalenza delle parole piane in italiano, sarebbe sensato includere quest'informazione nell'introduzione e indicare l'accento in 11 parole ultimali, per es. BLU (blü), LUNEDÌ (lunedi) $^{17}$, e 37 parole terzultimali, per es. GOMITO (gomito), ASINO (azino).

In 1000IB, invece, l'accento è segnalato in quasi tutti i casi, ma spesso in modo incoerente ed errato (per i punti critici si veda la tab. 10). L'accento manca 10 volte, mentre potrebbe essere considerato superfluo in 52 casi - si tratta perlopiù di monosillabi atoni quali articoli, congiunzioni, preposizioni (cfr. Canepari, 1999, p. 150; Maturi, 2006, pp. 95-96; Graffi e Scalise, 2008, p. 84) ${ }^{18}$, meno frequentemente di parole polisillabiche (composte o semplici). In ben 77 casi, invece, è scorretta la posizione dell'accento - nella maggioranza dei casi (49) si tratta della posizione davanti al nesso $\mathrm{s} / \mathrm{z}+$ consonante (l'accento dovrebbe seguire $\mathrm{s} / \mathrm{z}$ a causa della sillabazione quando il nesso è preceduto da una vocale, cfr. /les-'ka-le/ le scale; Canepari, 2006b, p. 125); piuttosto simili sono 15 casi in cui la posizione dell'accento è scorretta, benché si riferisca alla sillaba corretta; più problematica è invece l'accentazione di una sillaba errata (13). 4 trascrizioni includono un segno errato: gli accenti grave (3) e acuto (1).

${ }^{16}$ L'italiano è una lingua ad accento dinamico (Nespor, 1993, p. 65), mentre lo sloveno (anche standard) conosce sia l'accento dinamico sia l'accento musicale (l'ultimo non è caratteristico di tutti i dialetti; Toporišič, 2004, p. 63).

${ }^{17} \mathrm{Nel}$ caso delle parole ultimali con l'accento grafico e parole ultimali monosillabiche si potrebbe evitare la segnalazione dell'accento. La sua segnalazione in questi casi sarebbe giustificata dal principio di coerenza e dalla riduzione delle informazioni introduttive.

${ }^{18}$ In 1000IB i monosillabi atoni sono accentati o meno (la prima e la seconda cifra indicano il numero delle occorrenze accentate e non-accentate): preposizioni a 3/4, ad 1/0, da 2/13, di 8/16 (del $2 / 0$ ), con 1/0, in 1/0, per 1/0, su 1/1, tra 1/0; articoli il 0/1, le 9/0, un 4/0; congiunzione e 4/2. 
Tab. 10. Accento in 1000IB - punti critici

\begin{tabular}{|c|c|}
\hline accento mancante ${ }^{10}$ & 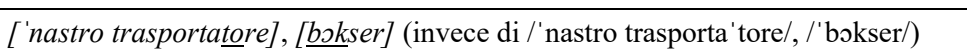 \\
\hline accento superfluo $^{52}$ & $\begin{array}{l}\text { ['fjokko 'di 'neve], ['ssno 'le 'stto] (invece di /'fjokko di 'neve/,/'sono le 'otto/) } \\
\text { ['video'kamera], ['giat'tfo] (invece di /video'kamera/, /'gjatto/) }\end{array}$ \\
\hline posiz & 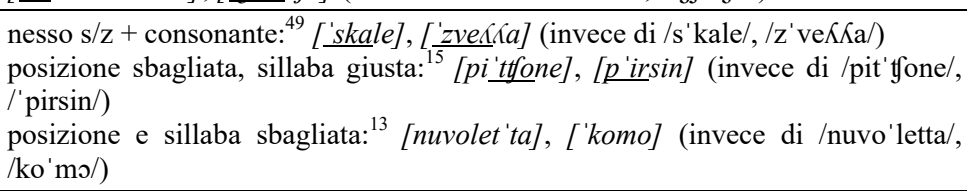 \\
\hline segno sbagliato ${ }^{4}$ & 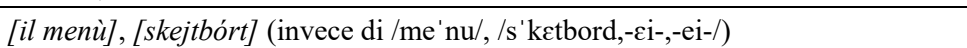 \\
\hline
\end{tabular}

\section{DISCUSSIONE E CONCLUSIONE}

Lo scopo del presente contributo è stata l'analisi della presentazione della pronuncia italiana in cinque dizionari per bambini e ragazzi sloveni. Dato che si tratta di utenti particolari (a causa della loro età, delle loro capacità, conoscenze, necessità), bisogna essere molto prudenti nella presentazione degli aspetti foneticofonologici nei dizionari che si rivolgono a loro.

Il primo contatto con la pronuncia in un dizionario avviene con le osservazioni fonetico-fonologiche introduttive, che sono presenti in misura limitata in tre dizionari analizzati - SISS contiene l'alfabeto italiano con i fonemi corrispondenti (trascritti con l'AFI), OSS presenta una breve nota (per i genitori) sulla trascrizione semplificata (con l'alfabeto sloveno) e l'alfabeto italiano (con esempi illustrativi), mentre 1000IB include una nota più lunga sul valore fonetico di alcuni grafemi e gruppi grafemici italiani. In tutti i dizionari analizzati (ma soprattutto nei dizionari senza la trascrizione) sarebbe necessaria la presentazione del rapporto grafia-pronuncia (si potrebbero menzionare inoltre alcuni elementi fonologici tipici, si veda avanti), mentre 1000IB dovrebbe contenere anche una spiegazione della trascrizione AFI.

Solo due dizionari includono la pronuncia di tutti i lemmi - la trascrizione fonologica in OSS si basa sull'alfabeto sloveno, in 1000IB sull'AFI (in SISS sono trascritte con l'AFI le lettere dell'alfabeto italiano). Il punto problematico della prima trascrizione è la sua approssimatività (alcune lettere corrispondono a più fonemi, le palatali italiane sono troppo semplificate) e l'assenza dell'accento. Il difetto più grande della trascrizione in 1000IB è invece l'assenza di una nota esplicativa sul rapporto tra l'AFI e l'alfabeto sloveno (per es. $/ \mathrm{t} /$ corrisponde alla pronuncia della lettera 〈č〉 in čas 'tempo') che informerebbe l'utente sul valore dei simboli usati (altrimenti l'utente deve essere a conoscenza dell'AFI o informarsi sull'AFI altrove); altri punti deboli sono la presenza degli allofoni nasali e le parentesi quadre (invece delle barre oblique).

Per quel che riguarda la trascrizione dei singoli fonemi in OSS, sono problematiche particolarmente le vocali medie $/ \varepsilon \mathrm{o} /$ e le consonanti $/ \kappa \mathrm{n} \mathrm{w} /$, trascritte 
sempre come (e), (o), (u v), (lj) e (nj), e /j dz/ spesso rappresentate da (i) e (c); la trascrizione delle vocali /i a/ e delle consonanti /ts $\mathrm{z}$ v/ presenta meno errori. In $1000 \mathrm{IB}$ è problematica la trascrizione delle vocali medie (cfr. la confusione tra /e $\varepsilon /$ e /o o/) e degli allofoni nasali [ $\mathrm{n} \mathrm{m}$ n,], gli altri errori di trascrizione, riguardanti le vocali /i u/ e le consonanti / $\mathrm{n} \mathrm{d} \mathrm{dz} \mathfrak{y} \mathrm{s} \mathrm{z} \int 3 \mathrm{j}$ w/, sono per lo più sporadici. In OSS sarebbero opportuni, innanzittutto, segni speciali per $\mathrm{i}$ fonemi $/ \varepsilon \mathrm{o} / \mathrm{e} / \mathrm{N}_{\mathrm{n}} \mathrm{w} /$ (accompagnati preferibilmente da un commento introduttivo) ${ }^{19}$, mentre la trascrizione in 1000IB dovrebbe trascurare la variazione allofonica. La trascrizione in entrambi i dizionari dovrebbe essere più precisa e coerente (basata su una delle varianti di pronuncia più accettabili, cfr. le pronunce moderna e tradizionale; Canepari, 1999).

In OSS, le consonanti lunghe più problematiche, perché sempre trascritte come semplici, sono $/ \mathfrak{t} d \mathrm{~d} \int \mathrm{n} K /$, meno problematiche sono $/ \mathrm{k}$ ts/ lunghe. Tra le consonanti lunghe spesso trascritte come semplici in 1000IB bisogna menzionare soprattutto /d ts $\mathrm{J}$, meno errori - riguardanti la durata - caratterizzano le trascrizioni di $/ \mathrm{b} \mathrm{k}$ $\mathrm{d} \mathrm{d} \mathrm{s} 1 \mathrm{n} /$.

L'accento in OSS è indicato solo una volta, in una parola piana. In 1000IB, invece, l'accento fa parte delle trascrizioni fonologiche, ma è spesso errato: in alcuni casi l'accento manca o è superfluo (in monosillabi atoni, meno frequentemente in parole polisillabiche); più spesso l'accento si trova in una posizione scorretta (problematici sono soprattutto i casi in cui l'accento si riferisce a una sillaba errata); qualche volta è usato un segno accentuale sbagliato. In OSS sarebbe più ragionevole indicare l'accento in tutte le parole ultimali e terzultimali, menzionando - nell'introduzione - che le parole rimanenti (i.e. la maggior parte delle parole italiane) sono piane. In 1000IB invece dovrebbero essere segnati, correttamente, tutti gli accenti primari (tranne in monosillabi atoni; cfr. Canepari, 1999, p. 150; Graffi e Scalise, 2008, p. 84), mentre gli accenti secondari (cfr. ['video'kamera] con l'accento secondario segnato in modo errato) non sono distintivi (Canepari, 1999, p. 153; Bertinetto, 2010, p. 16) ${ }^{20}$.

Tra i dizionari analizzati solo PIB include registrazioni audio che rappresentano, ovviamente, il mezzo più diretto per accedere alla pronuncia corretta delle parole

\footnotetext{
${ }^{19}$ Il fonema meno problematico, per quel che riguarda la trascrizione, è probabilmente $/ \mathrm{w} /$, cfr. la lettera (w) nota al pubblico sloveno grazie (soprattutto) all'alfabeto inglese. Due soluzioni per le consonanti / $\varepsilon \supset \kappa \mathrm{n} /$ potrebbero essere invece lettere barrate, per es. (e $\theta \mathrm{n}$ ), o segni diacritici, per es. (è ò n' l'). Entrambe le possibilità hanno dei punti deboli, ma dato che la nota sulla pronuncia (cfr. OSS, p. 2) si rivolge ai genitori, questi potrebbero agire da mediatori nella comprensione delle diverse possibilità.

${ }^{20} \mathrm{La}$ trascrizione fonetica sarebbe molto più complessa, perché dovrebbe tenere conto anche degli allofoni, della durata vocalica, dei gruppi accentuali, della cogeminazione, ecc. (Canepari, 1999), cfr. [, sakkoap'pe:lo], [, sonole, sEtteed'dje:tfi.] o [, sonole'set'te- ed'dje:fí.] invece di ['sakko 'a 'pelo], ['sono 'le 'sctte 'e 'djetii].
} 
italiane. PIB, purtroppo, non include delle osservazioni sul rapporto tra la pronuncia e la grafia, che potrebbero avere un valore più generale.

Il dizionario per bambini e ragazzi è uno dei mezzi con cui un giovane apprendente può cominciare a imparare una L2/LS, osservando le immagini con l'insegnante, il genitore, guardando, ascoltando e pronunciando le parole scritte in esso. Per arrivare, tramite un dizionario, alla dimensione orale di una L2/LS l'apprendente (o l'utente del dizionario) deve disporre di alcuni elementi: registrazioni audio, osservazioni sul rapporto grafia-pronuncia o sulle caratteristiche fonetico-fonologiche L2/LS, trascrizioni comprensibili e corrette, precedute da qualche nota introduttiva. Senza questi elementi (e presupponendo che l'utente non abbia conoscenze precedenti) arrivare alla pronuncia L2/LS sembra difficile. I cinque dizionari analizzati nel presente contributo risultano frammentari, per quel che riguarda il loro aspetto fonetico-fonologico - solo uno include registrazioni audio, ma non offre una guida alla pronuncia (al rapporto grafia-pronuncia), due includono trascrizioni fonologiche lacunose e note sulla pronuncia insufficienti, uno dispone delle lettere italiane con i fonemi corrispondenti, mentre un altro è privo di qualsiasi elemento che possa avvicinare l'apprendente all'aspetto fonetico-fonologico della lingua italiana. Per quel che riguarda la pronuncia italiana, dunque, i dizionari per bambini e ragazzi sloveni lasciano alquanto a desiderare. 


\section{BIBLIOGRAFIA}

Ahmad, K. (2016). Integrating Pronunciation with Vocabulary Skills. In T. Jones, J.B. Gilbert (eds.), Pronunciation in the Classroom: The Overlooked Essential (pp. 1-16). Alexandria (VA): Tesol Press.

Bartolí Rigol, M. (2005). La pronunciación en la clase de lenguas extranjeras. Phonica, 1, 1-27.

Bertinetto, P.M. (2010). Fonetica italiana. Quaderni del Laboratorio di Linguistica della Scuola Normale Superiore, 9/1, 1-30.

Brecelj, K.K. (2013). Difficoltà di pronuncia dei parlanti sloveni nell'apprendimento della lingua italiana. Vestnik za tuje jezike, 5/1-2, 183-199.

Canepari, L. (1999). Manuale di Pronuncia Italiana. Bologna: Zanichelli.

Canepari, L. (2006a). Pronunce straniere dell'italiano. München: Lincom Europa.

Canepari, L. (2006b). Avviamento alla fonetica. Torino: Einaudi.

Canepari, L. (2012). Dipi: dizionario di pronuncia italiana online. Retrieved September 30, 2019 from http://www.dipionline.it/dizionario.

Costamagna, L. (2000). Insegnare e imparare la fonetica. Torino: Paravia.

Dall'Armellina, R., Turolla, M.L., Gori, G. (2005). Giocare con la fonetica. Firenze: Alma Edizioni.

Fraser, H. (1996). Pronunciation guides for children. The Australian Journal of Language and Literacy, 19 (3), 221-243.

Freudstein, R. (1995). Insegnare le lingue straniere ai bambini. Roma: Armando.

Godec Soršak, L. (2015). Before compiling a primary school dictionary. SKY Journal of Linguistics, 28, 161-186.

Grad, A. (1991). Italijansko-slovenski in slovensko-italijanski slovar. Ljubljana: CZ.

Graffi, G., Scalise, S. (2008). Le lingue e il linguaggio: introduzione alla linguistica. Bologna: il Mulino.

Greenberg, M.L. (1987). Prozodične možnosti v slovenskem knjižnem jeziku. Slavistična revija, 35/2, 171-186.

Grošelj, R. (2013). Vocali a confronto: analisi contrastiva dei sistemi vocalici sloveno e italiano. Études romanes de Brno, 34/2, 131-147.

Heinz, M. (ed.) (2009). Le dictionnaire maître de langue. Lexicographie et didactique. Berlin: Frank \& Timme.

Jones, T. (2016). The Gap between the Integration of Pronounciation and Real Teaching Contexts. In T. Jones, J. B. Gilbert (eds.), Pronunciation in the Classroom: The Overlooked Essential (pp. XI-XXI). Alexandria (VA): Tesol Press.

Maturi, P. (2006). I suoni delle lingue, $i$ suoni dell'italiano. Nuova introduzione alla fonetica. Bologna: il Mulino.

Mikhailov, N. (1997). Dizionario sloveno: italiano-sloveno, sloveno-italiano. Milano: Antonio Vallardi.

Mioni, A.M. (1993). Fonetica e fonologia. In A.A. Sobrero (ed.), Introduzione all'italiano contemporaneo. Le strutture (pp. 101-139). Roma/Bari: Laterza.

Nespor, M. (1993). Fonologia. Bologna: il Mulino.

Palermo, M. (2015). Linguistica italiana. Bologna: il Mulino.

Parnwell, E.C. (1988). The New Oxford Picture Dictionary. New York/Oxford: OUP.

Pointon, G. (2016). The Representation of Pronunciation in General Dictionaries. In P. Durkin (ed.), The Oxford Handbook of Lexicography (pp. 472-487). Oxford: Oxford University Press.

Rivera González, M.D.R. (2005). ¿Se puede enseñar fonética con un diccionario? In Las gramáticas y los diccionarios en la enseñanza del español como segunda lengua: deseo 
y realidad. XV Congreso Internacional de la ASELE, Sevilla, 2004 (pp. 733-740). Sevilla: Universidad de Sevilla.

Schmidt, S., Pedrazzini, G. (2016). La pronuncia delle occlusive nel tedesco L2 di apprendenti italofoni: un esperimento didattico. In R. Savy, J. Alfano (eds.), La fonetica nell'apprendimento delle lingue (pp. 45-60). Milano: AISV.

Sisinni, B. (2016). Fonetica e fonologia della seconda lingua. Roma: Carocci.

Skela, J. (1999). Glasovna podoba jezika (fonologija). In L. Čok, J. Skela, B. Kogoj, C. Razdevšek Pučko (eds.), Učenje in poučevanje tujega jezika (pp. 122-126). Koper: Znanstveno-raziskovalno središče Republike Slovenije; Ljubljana: Pedagoška fakulteta.

Šlenc, S. (1997). Veliki italijansko-slovenski slovar. Ljubljana: DZS.

Tivadar, H., Jurgec, P. (2003). Podoba govorjenega slovenskega knjižnega jezika v Slovenskem pravopisu 2001. Slavistična revija, 51/2, 203-220.

Toporišič, J. (1992). Enciklopedija slovenskega jezika. Ljubljana: Cankarjeva založba.

Toporišič, J. (2004). Slovenska slovnica. Maribor: Obzorja.

Torres Martínez, M. (2017). Enrédate. Diccionario temático para niños: explotación en el ámbito de ELE. E-Aesla. Revista Digital, 4, 428-436.

Torresan, P. (2010). Didattica per lo sviluppo della competenza fonetico-fonologica in lingua straniera. Entre lenguas, 15, 59-73.

Tritch Yoshida, M. (2016). Beyond Repeat After Me: Teaching Pronunciation to English Learners. Alexandria (VA): Tesol Press.

Villaescusa Illán, I. (2009). La enseñanza de la pronunciación en la clase de ELE. In N. Arriaga Agrelo (ed.), El currículo de E/LE en Asia-Pacífico. Selección de artículos del I congreso de español como lengua extranjera en Asia-Pacifico (CE/LEAP) (pp. 127-145). Manila: Instituto Cervantes, Embajada española.

Wells, J.C. (1985). English Pronunciation and its Dictionary Representation. In R. Ilson (ed.), Dictionaries, lexicography, and language learning (pp. 45-51). Oxford/New York/Toronto/ Sydney/Frankfurt: Pergamon Press, The British Council.

Yule, V. (1989). Children's dictionaries: spelling and pronunciation. English Today, 17/1, 13-17.

\section{Fonti}

1000IB = Ferrarová, M. (2014). 1000 italijanskih besed . Kranj: Narava.

1351BAINS = Bortolussi, L.A. (2003). 1351 besed $v$ angleščini, italijanščini, nemščini in slovenščini: štirijezični slikovni slovar za male in velike. Trad. M. Kraigher. Ljubljana: Alba 2000.

OSS = Flamini, L. (2014). Otroški slikovni slovar. Trad. M. Ozimek. Ljubljana: Grahovac.

PIB = Morris, N. (2011). Prve italijanske besede. Trad. L. Virnik Kovač. Kranj: Narava.

SISS = Majcenovič Kline, B. (2018). Slovensko-italijanski slikovni slovar. Hoče: Skrivnost. 